\title{
Lyndonizando a Barry: un zoom hegeliano
}

\section{Lyndonizing Barry: A Hegelian Zoom}

\author{
Alejandro Varas \\ Escuela de psicología, PUCV \\ alejandro.v.alvarado@gmail.com
}

\section{Resumen}

El presente ensayo realiza un análisis del film Barry Lyndon, dirigido por Stanley Kubrick, desde algunas nociones planteadas por G. W. F. Hegel en su obra Fenomenología del espíritu. En particular, se plantea cómo el "zoom in" en dicho film posibilita una mediación entre los yoes individuales de Redmond Barry, los cuales se articulan como momentos de un movimiento lógico subjetivo, que da cuenta del carácter negativo de la identidad. Para ello se analizan dos escenas específicas del film, estas son su escape del ejército inglés y la aparición de Lady Lyndon; se argumenta cómo, en cada una de ellas, el zoom propone un devenir Otro, el cual niega tanto al protagonista como al espectador del film.

Palabras clave: Barry Lyndon, zoom, mediación, movimiento, negatividad.

\section{Abstract}

The following essay it's an analysis of Stanley Kubrick's Barry Lyndon, from some notions proposed by G. W. F. Hegel in The Phenomenology of Spirit. Particularly, how the use of "zoom in" in the film makes possible mediation between Redmond Barry's individual "I"s, articulated as moments of a logical subjective movement, which accounts for the negative nature of the identity. For that purpose, two specific scenes of the film are analyzed, first, his escape from the British Army and Lady Lyndon's appearance. It is argued how, in each of them, the zoom proposes the becoming of Other, which denies both the protagonist and the spectator of the film.

Keywords: Barry Lyndon, Zoom, Mediation, Movement, Negativity. 
El éxito de Hegel marcó el comienzo de la «edad de la deshonestidad" $[. .$.$] y de la «edad de la irresponsabilidad» [. .$.$] :$ el comienzo de una nueva edad controlada por la magia de las palabras altisonantes y el irresistible poder de la jerigonza.

Karl Popper

En esencia, es una historia tan poco original que Stanley Kubrick no hizo muchas cosas impresionantes con su cámara para contarla; podría haber sido mucho más impactante. Así y todo, necesita esforzarse para mantener nuestro interés en una historia que nos ha sido contada ya innumerables veces.

Crítica en prensa a Barry Lyndon

Hegel y Kubrick. Sujetos de desprecio a la vez que de alabanza. ¡Cuánta tinta en el papel sobre la obra del filósofo alemán y cuánta columna de crítica cinematográfica en torno al director estadounidense! Cada uno de ellos, ya sea por el profundo rechazo generado o por la admiración que provoca en sus seguidores, marca un momento en la historia de la filosofía y el cine. Ambición megalómana, obsesión puntillista, y un deseo voraz por comprender y crear nuestro mundo. John Alcott, director de fotografía de Kubrick, comentó alguna vez: "Stanley Kubrick no simplemente crea films, él crea mundos enteros en ellos" (American Cinematographer 269). Esto no se traduce en la forja de un millar desperdigado de producciones. Al contrario, nos hallamos -en ambos casos- ante un número acotado de obras que reflejan un afán por sistematizar el todo, con detalle, precisión, astucia y belleza. Dicho afán no adviene de modo inmediato, sino que se encuentra mediado por la paciencia, la insistencia y el análisis que podamos dedicar a la contemplación y comprensión de dichas obras. En Hegel, dos escritos infinitos: La Fenomenología del espíritu (1807) y la Ciencia de la Lógica (1812). En Kubrick, una filmografía en la que destacan películas como Lolita (1962), 2001: Odisea del espacio (1968), La naranja mecánica (1971), El resplandor (1987) y Ojos bien cerrados (1999), entre otras. Respecto a dichos films, destaca uno en especial por su belleza y precisión, del cual Kubrick mencionó antes de su realización: "A los 45 años es ya tiempo de haber rodado mi obra maestra". Pese a ello, tanto por el público como por los críticos de cine de su tiempo, fue calificada de aburrida, larga y poco original. Hablamos de Barry Lyndon (1975). A través del análisis de esta película, colocaremos en diálogo a Kubrick con Hegel, enhebrando a ella algunas ideas conducentes a una lectura hegeliana de la misma. ¿Qué Hegel nos muestra Kubrick? ¿Qué dimensión hegeliana nos expresa Barry Lyndon? Ese es nuestro camino. 


\section{Un zoom a Barry Lyndon}

Barry Lyndon es una película que Kubrick decide realizar luego de desechar la idea de filmar una en torno a Napoleón Bonaparte. Apasionado por el dictador y su historia, Kubrick había leído sobre aquel e incluso había redactado un guión. Sin embargo, estando en la etapa de preproducción, apareció en la pantalla grande Waterloo (1970), de Sergei Bondarchuk, lo que transformó en una muy mala idea comercial aquella intención. Sin embargo, el interés de grabar algo en relación a tal contexto epocal no es abandonado por Kubrick, quien luego de aquel imprevisto decide llevar al cine la novela de William Makepeace Thackeray llamada The Luck of Barry Lyndon (1844). El largometraje nos muestra la historia de Redmond Barry, un joven irlandés de origen humilde, a finales del siglo XVIII que, dado su arrojo frente a la vida, vive múltiples experiencias en diversos contextos sociales. En ese camino, Redmond logra, no sin obstáculos, ascender en la escala social, económica y cultural de su época, logrando casarse con una viuda de gran belleza y riqueza, llamada Lady Lyndon, mediante quien consigue su apellido. Un desenlace trágico termina por dejar a Redmond abandonado en el desprecio, la ruina y una amputación que lo deja inválido.

El film fue nominado a 7 óscares: mejor película, mejor director, mejor guión adaptado, mejor fotografía, mejor dirección artística, mejor banda sonora, y mejor diseño de vestuario. De aquellos óscares, obtuvo todos menos los dos primeros. Esto decepcionó a Kubrick, sumando a ello no sólo la poco benévola crítica cinematográfica, sino además el hecho de que logró recaudar apenas 9,5 millones de dólares de los 30 esperados por Warner, teniendo en cuenta que el film costó alrededor de 11. Sin embargo, el tiempo le daría la razón a Kubrick y actualmente es considerada una de las mejores películas de época y una de las más grandes producciones de dicho director. El uso de la luz, la composición, el vestuario y la música, entre otros elementos, la transforman en una verdadera obra de arte. Famosa es la historia de cómo Kubrick consiguió iluminar los espacios interiores mediante velas encendidas y ventanales abiertos; ello fue posible en gran parte al cruzar astutamente a unas cámaras Mitchell BNC las lentes Carl Zeiss de f0.7 usadas por la NASA. ¿El resultado? Una cámara que fue capaz de recoger como nunca antes la sutil iluminación propia de la época en donde los personajes vivieron.

La utilización del zoom en este film merece atención aparte. Esta técnica ha sido demonizada en el cine por su uso desmesurado. Su empleo en Barry Lyndon ha sido analizado desde el efecto que genera: "la bidimensionalidad y planitud de la imagen" (Falsetto 59). Sin embargo, Kubrick logra mediante aquel efecto otorgar a su film una atmósfera estética y epocal, como si las escenas fuesen cada una un óleo del siglo XVIII. Como comenta John Alcott: "cada toma es un cuadro en sí" (Ciment, Kubrick: edición definitiva, 216). Esta tesis sobre el uso del zoom es compartida en diversos análisis realizados en torno al film; por ejemplo, el director Martin Scorsese señala: 
Yo sabía que Barry Lyndon era un film de época, pero esperaba algo diferente. Y la llevó al pasado. El uso del zoom es interesante porque nunca piensas en usar zoom en el pasado. El zoom aplana la imagen como en una pintura del XVIII (cit. en Harlan).

Sobre esta técnica cinematográfica deseamos detenernos. Nuestra intención es examinar dicho zoom desde una óptica filosófica, la cual puede llevarnos a un modo alternativo de comprender el film. ¿Es posible una interpretación diferente del uso de aquel zoom? ¿Un análisis más allá de su dimensión estética, pictórica, o fílmica? ¿Una comprensión filosófica de Barry Lyndon desde la operación que realiza dicho zoom? Con motivo de aquel propósito invitaremos a Hegel, teniendo desde ya en mente algunos puntos de anclaje entre lo propuesto por el cineasta y lo planteado por el filósofo. Y resulta a lo menos curioso que, en el cruce de estos dos maestros, Barry Lyndon no sólo aparezca como un hijo de Kubrick, sino también como el retrato de una época en la que el mismo Hegel vivió, período de guerras y revoluciones, de dictaduras y prosperidad intelectual. Aún más: la historia de Redmond podría no resultar tan lejana a la de un desconocido filósofo pueblerino que, mediante su gran talento y sus influyentes amistades, logró transformarse en rector de la Universidad de Berlín. ¿Qué puede Barry Lyndon contarnos sobre Hegel?: un contexto sociohistórico que permite la emergencia de la filosofía hegeliana. ¿Qué puede Hegel contarnos sobre Barry Lyndon?: algunos planteamientos del filósofo, planteados en su Fenomenología, nos ayudarán en dicha tarea.

\section{Superando la escisión: la Fenomenología del espíritu}

La Fenomenología del espíritu, una de las principales obras de Hegel, es escrita durante 1806 en Jena, mismo año en el que Bonaparte derrotará al Imperio Austriaco, Prusiano y Ruso juntos. La batalla de Austerlitz es recordada como la mayor victoria de Napoleón en las guerras por el control europeo bajo el Imperio Francés y significó la disolución del Sacro Imperio Romano Germánico, que poseía alrededor de mil años de antigüedad. Sólo unos meses después, en la mismísima ciudad de Jena, recoveco intelectual de Goethe, Schelling, Fichte, Hölderlin, Novalis, y Schlegel, entre otros, el Emperador francés vencerá definitivamente a Prusia, siendo posible posteriormente su entrada triunfal en Berlín. Hegel, en Jena, llegará a decir:

\footnotetext{
He visto al emperador - esa alma del mundo- salir de la ciudad para efectuar un reconocimiento. Es efectivamente una sensación maravillosa el ver a semejante hombre que, concentrado aquí en un punto, montado en su caballo, se extiende sobre el mundo y lo domina (Hoffmeister, cit. en Voegelin 176).
}

La filosofía de Hegel emerge en un período histórico convulsivo, época de crisis y transición; podríamos decir, en un período revolucionario. En su vida Hegel ya ha asistido a la Revolución de Estados Unidos, la Revolución Francesa, el Reino del Te- 
rror, y la autocoronación de Napoleón como Emperador. Posteriormente alcanzará a ser coetáneo de las Revoluciones Española e Hispanoamericanas, la Restauración del absolutismo en Europa, e incluso el inicio de la Revolución Industrial. Estas décadas, situadas al final de un siglo y al inicio de otro, dan cuenta de las grandes y efervescentes transformaciones sociopolíticas que dibujarán de manera importante el mapa de nuestro mundo actual.

Es en este contexto histórico político en el que se inserta Hegel. Un mundo en donde dichas transformaciones son expresión de un violento encuentro entre las ideologías protagonistas de la época y, en gran medida, entre los paradigmas filosóficos de la historia de occidente. Hegel es consciente de dicho período, de cómo una Aufklärung en declive convive con una emergente Sturm und Drang, en donde un cuadro de David o Ingres deviene un Goya o un Gericault, en donde una Enciclopedia observa horrorizada un río Sena bañado en sangre: una escisión impregna con su aroma las conversaciones, decisiones y reflexiones de la época. La filosofía de la escisión ha separado al hombre de su mundo, al sujeto del objeto, al creyente de Dios, y al artista de la belleza. Hegel, si bien criticará aquella escisión, dicha autoconsciencia devendrá fruto de un proceso de tránsito en tales escenarios escindidos, será una búsqueda llena de dubitaciones en donde Hegel ocupará por varios años ciertos territorios para luego abandonarlos y superarlos, construyendo sus propios. Aquel merodeo de treinta y seis años podemos seguirlo de cerca en sus Escritos de Juventud, en la correspondencia que va sosteniendo con sus amigos y compañeros del seminario de Tubinga, Friedrich Hölderlin y Friedrich Schelling. La escisión es compartida, dialogada y poetizada. Sólo luego de la muerte de su padre, al finalizar el siglo XVIII, Hegel declarará su maduración intelectual. La Fenomenología aparece como ícono de aquel momento de diferenciación. Hegel se alza observando, analizando y superando dicha escisión filosófica y las contradicciones históricas que se despliegan desde ella; deja de una vez y para siempre a sus maestros para caminar por sí mismo mediante lo lógico. Las antinomias de la razón en Kant, el germen de un yo totalitario en Fichte, la poesía como fundamento en Hölderlin y la naturaleza indescifrable en Schelling, serán acusadas en su Fenomenología como escisiones a superar.

Fenomenología del espíritu es un título escogido por Hegel luego de ambivalencias ante otros posibles como Ciencia de la experiencia de la consciencia, o Ciencia de la fenomenología del espíritu ( Fenomenología del espíritu 945). Estos otros títulos no sólo nos aclaran una pretensión de otorgar un estatuto científico a la filosofía, que para Hegel no es más que "amor por el saber", sino que además dejan en claro, ya en su inicio, la lógica inserta en las páginas de dicha obra. Hablar de una fenomenología del espíritu es hablar de dos dimensiones escindidas en la historia de la filosofía: por un lado el fenómeno, por otro el noúmeno; por un lado la cosa, por otro el sujeto; por un lado la objetividad, por otro la subjetividad; por un lado lo empírico, por otro lado lo racional. El afán aquí es mediar entre aquellos dos mundos, articulándolos de manera radical; hablar de una fenomenología del espíritu es hablar de una cons- 
ciencia fenoménica, de un sujeto cósico, de una subjetividad objetiva, de lo empírico racional. Esta superación de la escisión propone la mediación como operación lógica que constituye al mundo en virtud de su negatividad, es decir, en relación a lo que éste no es. La mediación opera como la articulación que conecta el movimiento del ser al no ser, que enfrenta e inserta la negación como momento no contradictorio, sino inherente al sistema. Sobre esto Hegel insistirá a lo largo de toda su Fenomenología; podemos leer ya en su primer capítulo:

\footnotetext{
Ahora; ya ha dejado de ser en cuanto se lo muestra; el ahora que es, es otro que lo mostrado, y vemos que el ahora es precisamente esto: en tanto que es, ya ha dejado de ser. El ahora, según nos es mostrado, es algo sido; y ésta es su verdad; no tiene la verdad del ser. Lo verdadero es, pues, que ha sido. Pero lo que ha sido no es, de hecho, esencia alguna; no ES, y de lo que se trataba era del ser (171).
}

Sin embargo dicha esencia o verdad no se acaba en la negación, sino en la negación de una negación que mediatiza los elementos negados. Es el momento en que la concepción del mundo no acaba en su momento primero, o en su momento final, sino más bien en ambos como distintos momentos que expresan diversas verdades sobre el mundo. Como plantea Hegel en el Prefacio: "la cosa no queda agotada en su fin, sino en su desenvolvimiento y ejecución, y tampoco el resultado es el todo real, sino que el todo real es el resultado junto con su devenir" (113). Es aquel desenvolvimiento y ejecución como devenir, aquella lógica, mediación y negatividad, aquella superación de la escisión con la que proponemos insertarnos en la vida de Redmond Barry, esta vez contada en imágenes por Stanley Kubrick.

\section{Lyndonizando a Barry}

¿Qué puede significar algo así como Lyndonizar a Barry? Implica mostrar cómo en el proceso de transformación del protagonista a lo largo del film es posible escuchar el susurro de una lógica o un método. Lógica o método que nos habla sobre el ser, sobre la realidad, sobre el yo. Un yo singular, un Redmond que, en su historia, se transforma a sí mismo en otro yo, en un Lyndon. Y cómo esta transformación nos habla del devenir, de la mediación, de la negatividad y de la libertad: Hegelizando a Kubrick. El análisis de dos escenas del film se muestra especialmente atingente para tal tarea, escenas en las cuales el papel del zoom es central, las cuales articulan lo cinematográfico con lo filosófico. Estas son: la escena de los homosexuales en el lago y la escena de la aparición de Lady Lyndon.

En la primera escena, Redmond descubre la relación amorosa entre el Teniente Jonathan y su pareja, de nombre Frederick, y, fruto de ello, encuentra la oportunidad para escapar del ejército. El protagonista se encuentra alistado en la milicia inglesa desde hacía ya varios años y, a esas alturas, con intenciones francas de desertar lo más 
pronto posible. La miserable vida militar junto a la desoladora pérdida de su tío en la batalla ya eran suficientes razones para tomar una decisión así. Redmond observa que Jonathan habla sobre la inevitable tarea que nuevamente le han encomendado; esta consiste en llevar unos despachos reales con urgencia, lo cual entristece mucho a Frederick. A la vez, Redmond toma consciencia que la ropa, la documentación y el caballo de Jonathan se encuentran junto al árbol desde el cual observa a escondidas. Sin pensarlo mucho, Redmond toma al animal y dichos objetos, y deja a la pareja discutiendo en el lago sin que ésta se percate de la suplantación de identidad que le permite desertar.

El zoom muestra, en el plano inicial (figura 1), a Barry de espaldas a la cámara, con su cabeza en el centro, mirando hacia el lago que está al fondo. Justo delante de Barry, el tronco de un gran árbol que le sirve de escondite . La pareja, en el plano inicial, aparece muy cerca del centro, un poco más abajo y a la derecha, señalando ya el destino del zoom y el plano final. En el tronco del árbol se encuentra la ropa del Teniente y, a la izquierda de Barry, el caballo. El zoom viaja desde este plano, colocando al espectador en el lugar mismo de Barry, emergiendo así el plano final que muestra de cerca a la pareja en el lago (figura 2).

La segunda escena nos muestra a un Redmond ya asentado en un estilo de vida propio de la alta sociedad, de refinados gustos, costosos vestuarios y ornados ambientes. La compañía del Chevalier de Balibari le trae buenos dividendos, accediendo así a selectos círculos nobles, codeándose incluso con importantes figuras de la realeza. Dicha posición social, sin embargo, no se acompaña del estatus y la estabilidad deseados que le permitan proyectarse hacia el futuro e independizarse como un noble. Llega a la conclusión en dicha etapa de su vida, ya sin ilusiones sobre el amor, sobre la necesidad de encontrar una esposa de fortuna suficiente y posición social adecuada. La escena nos muestra entonces a Redmond junto al Chevalier, sentados frente a una mesa, tomando el té junto a unos bocadillos. Ambos descansan en una terraza que da vista a un patio de hermosos jardines y con una pileta, situado en las inmediaciones de un palacio. En dicho patio, a lo lejos, aparece Lady Lyndon, caminando junto a su esposo Sir Charles Lyndon, su hijo Lord Bullingdon, y el Reverendo Runt. Habiendo fijado su mirada en ella, el propósito de Redmond ahora ya se encarnaba en una mujer en particular.

El plano inicial con el que comienza el zoom (figura 3) nos muestra de cerca a Redmond y al Chevalier, ambos de perfil, sentados de frente, y en medio de ellos la mesa en donde toman el té. A lo lejos, más allá de la baranda de la terraza, aparece la pileta en el centro, y en rededor de ella los jardines del patio. En éste, se ven algunas personas caminando lentamente. Desde allí Barry gira su cabeza hacia el fondo, y le sigue sólo un poco después el Chevalier. El zoom avanza por en medio de ambos, siguiendo nuevamente la mirada del protagonista, realizando esta "subjetiva" que sobrevuela la pileta y coloca en un primer plano a los Lyndon caminando (figura 4). El espectador visualiza a la condesa, quien destaca del grupo grandemente por el tipo y color de su vestuario, así mismo por su belleza. 


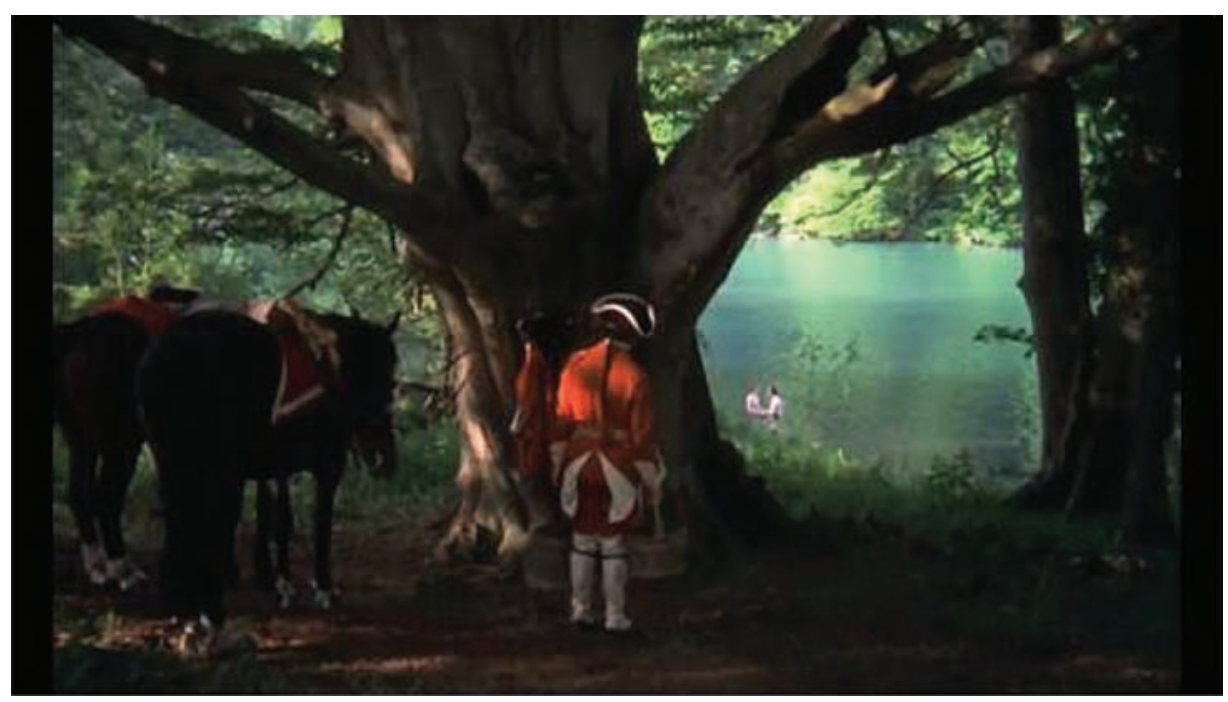

Figura 1. Redmond, escondido tras el árbol, descubre a la pareja de oficiales; el espectador es puesto detrás de la escena.

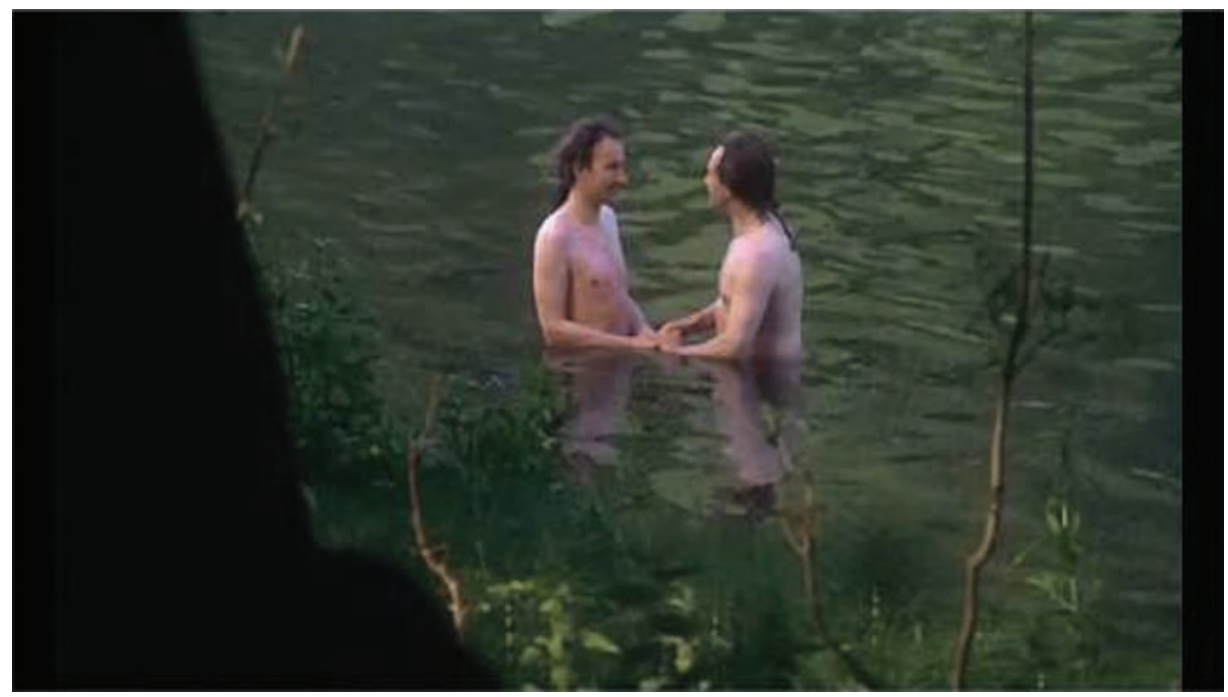

Figura 2. El zoom transforma a Barry en Fakenham, su mirada avanza junto con la cámara, a la vez que el espectador termina viendo con los ojos de Redmond.

Como mencionábamos, el zoom en este film ha sido motivo de varios análisis. Sin embargo, éste análisis ha puesto su énfasis en el zoom out, quedando relegado el papel que el zoom in juega. Esto llama absolutamente la atención, ya que ambos zooms descritos comparten una característica central a diferencia de los otros utilizados: son los dos únicos correspondientes a "subjetivas", las cuales llevan ese nombre en la medida en que otorgan al espectador una visión de lo que el protagonista se encuentra observando y, en este caso, lo colocan en el lugar de Redmond mismo. Considerando 


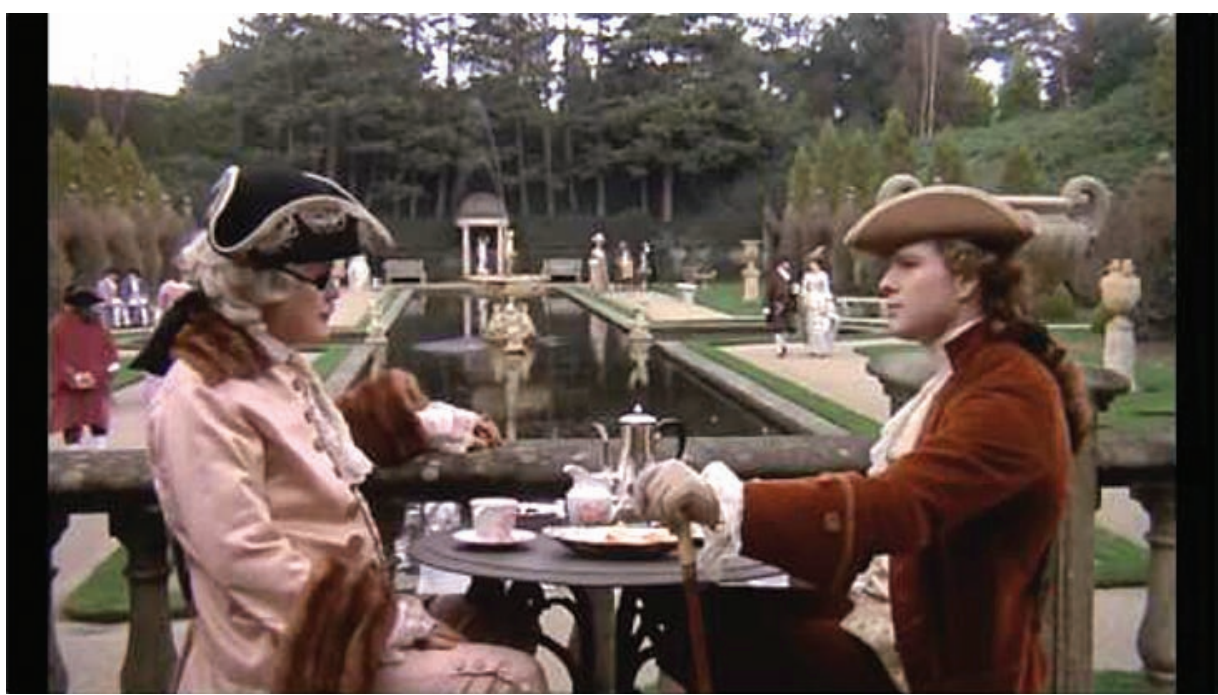

Figura 3. Redmond y el Chevalier toman el té, mientras desde el fondo aparece Lady Lyndon y compañía.

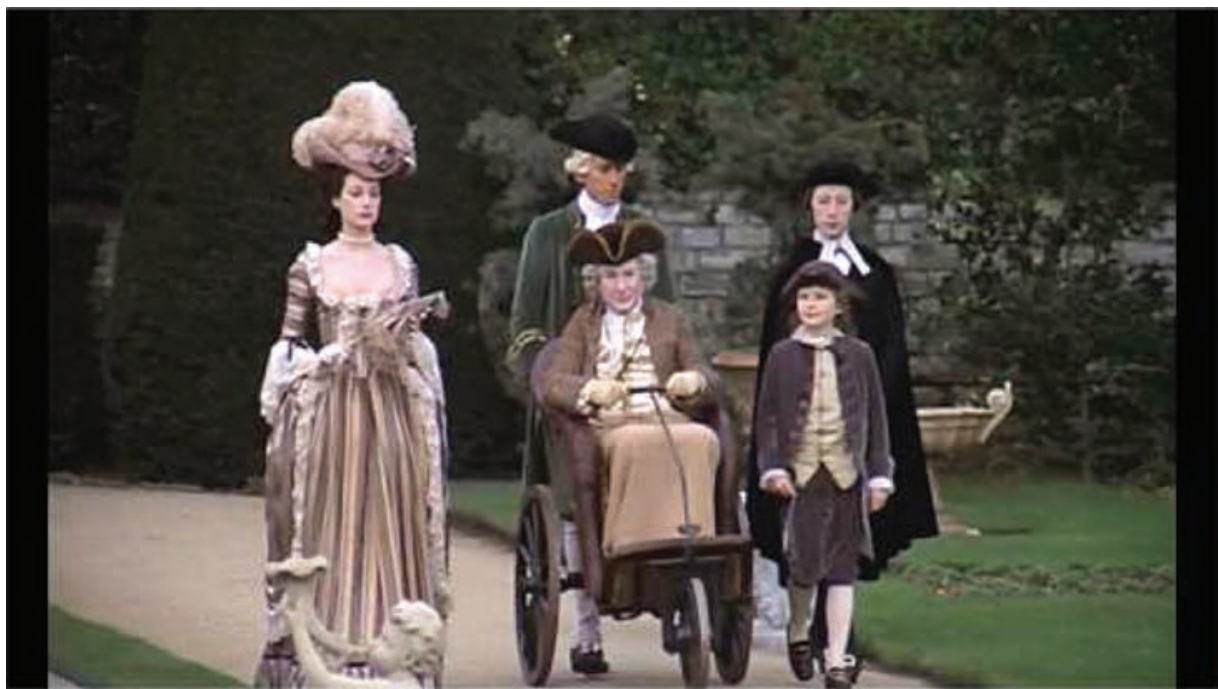

Figura 4. El zoom nuevamente expresa un pasaje, esta vez de Barry a Lyndon; el espectador -del mismo modo- termina observando desde la posición de Redmond.

que el uso de estos zooms no es azaroso por parte de Kubrick, ya desde la técnica debiésemos preguntar qué poseen de especial, para merecer este lugar en dichas escenas. Algo nos dicen sobre Redmond, algo nos dicen sobre la trama fílmica, algo nos dicen sobre lo que Kubrick busca retratar. ¿Qué nos puede decir el mismo Stanley Kubrick al respecto? En una entrevista realizada por Michel Ciment, el director profundiza en la realización de su película y en algunas claves interpretativas: 
Stanley Kubrick: - Barry Lyndon es una historia que no depende de la sorpresa. Lo importante no es lo que va a suceder, sino cómo va a suceder [...].

Michel Ciment:-Y la escena de los dos homosexuales en el lago tampoco estaba en el libro.

Stanley Kubrick: -El problema aquí era cómo conseguir que Barry saliese del ejército británico. La parte del libro que trata de esto es también bastante larga y complicada. La función de la escena entre los dos oficiales gays era proveer un modo más simple para que Barry escapase. Nuevamente, conlleva al mismo resultado final que en la novela, pero por un camino distinto. Barry roba los papeles y el uniforme de un oficial británico lo que le permite hacer su camino hacia la libertad. Como la escena es puramente expositiva, la situación cómica ayuda a enmascarar tus intenciones (Kubrick on Barry Lyndon: an interview with Michel Ciment).

Kubrick nos dice que lo que importa no es qué ocurrirá, sino cómo ocurrirá. El qué, el contenido, lo que Redmond y el espectador se encuentran observando, no son lo relevante. Es el cómo, es decir el modo, la forma, la manera en que suceden las cosas, lo que nos da luces sobre lo que Kubrick quiere relatarnos. ¿Qué cambio de enfoque supone pasar del qué al cómo? ¿Qué nos proponen estas escenas sobre el qué y sobre el cómo? El qué ya ha sido claramente expuesto, corresponde a la descripción del contenido de las escenas, de sus personajes, sus posiciones, los espacios en los que se encuentran, sus historias, pensamientos, deseos, y acciones. El qué son los sujetos y objetos planteados por Kubrick en los planos ya señalados. En este sentido, el cómo viene a ser la manera en que éstos sujetos y objetos son relacionados, articulados, puestos en movimiento, dinamizados. Vemos pues que el zoom usado en estas dos escenas nos habla de un cómo, de un modo particular en que emergen las figuras y cómo estas se van transformando en fondo, y viceversa. Kubrick nos habla de la función de la escena que permite a Barry robar y por tanto escapar, hacer su camino hacia la libertad. Y si tenemos por función en la primera escena un robar, un escapar, un ser libre, entonces no estamos tan lejos de la segunda. Redmond efectivamente en ésta última roba un nombre, escapa de su contexto social pretendiendo alcanzar su libertad llegando a otro más noble. Diremos entonces que este cómo, esta función, puede resumirse entonces en un movimiento.

¿Qué hay del cómo en estas escenas? En éstas no sólo se expresa el movimiento constante del protagonista a través de la sociedad, a través de los diversos estratos, capas o clases; aún más, expresan una lógica presente en aquel movimiento, a través del papel que poseen aquellos sujetos observados por Redmond en las escenas. Redmond vistiéndose de Jonathan se hace pasar por él, adoptando para sí su nombre; Redmond observando a Lady Lyndon ya anticipa su casamiento y la adopción del apellido de la condesa a su nombre propio. Redmond Barry se transforma en Jonathan Fakenham; Redmond Barry se transforma en Barry Lyndon. No se trata meramente de una suplantación de identidad ni del anexo de un apellido y un patrimonio, sino más bien del movimiento que el yo individual de Redmond realiza hacia otros yoes individuales- Jonathan 
Fakenham y Lady Lyndon- y cómo en ese movimiento Barry simultáneamente deja de ser quien para ser otro de sí mismo. De esta manera superamos la hipótesis de una mera suplantación de identidad como un robo astuto hecho al destino, y así también la referida al anexo del nombre de la condesa como frutos del escalamiento y la ambición del protagonista. En torno a esta primera hipótesis se mueve Martin Scorsese, al decir: "estás viendo el progreso de un hombre, de cómo él se mueve desde la inocencia más pura a la sofisticación más fría, terminando en la absoluta amargura - y es todo una cuestión de simple supervivencia" (cit. en Ciment, Kubrick: the definitive edition, viii). Podemos, en otro registro paralelo, dar cuenta que la trama nos habla de un yo en Redmond que deviene en otros yoes en la búsqueda de sí mismo y de su libertad. Mas, tampoco podemos admitir que estos movimientos provocan que Barry deje de ser lo que era realmente antes, o al contrario, de que se encuentre con quien realmente es en verdad en un futuro. Al contrario, Redmond es tanto ese yo individual como aquellos otros yoes individuales con los que se va fundiendo: ser con sus nombres involucra ser con sus yoes adjuntos, es decir, involucra inevitablemente ser esos otros yoes. Lischen, quien conoce a Redmond como Jonathan Fakenham, se despedirá de él, al final de su romance, diciendo "Goodbye, Redmond"; Lady Lyndon tratará a Barry con su nombre de pila, en la escena siguiente al matrimonio: "Redmond, would you mind not smoking for a while?”. Redmond siempre sigue siendo Redmond, a la vez que siempre va transformándose en otro de sí, en un Fakenham, en un Lyndon.

¿Y qué hay del cómo en estos zooms in? Es posible entonces ir más allá de la tesis tradicional sobre el uso de los zooms en este film, que propone que éstos permiten aplanar las escenas y hacerlas parecer así pinturas dieciochescas. El zoom aquí tratado es la expresión técnica de un movimiento, de un proceso en que el yo deviene. Entre Redmond y Jonathan, entre Redmond y Lady Lyndon hay una mirada que se dirige de un yo a otro yo, que implica tanto un movimiento del primero al segundo como una transformación del primero en el segundo. El zoom es lo que expresa la conexión y la transición de un lugar, de un momento a otro, el cual no contradice al anterior sino que lo engloba, complejizándolo, integrándolo, colocándolo en dinamismo y tensión. Como plantea Pliatska, al realizar un análisis filosófico del film, ahondando en el trabajo del absurdo existencial:

Una forma de observar el uso que Kubrick hace del zoom out es como una metáfora visual de las dos perspectivas que tomamos en nuestras vidas. Al empezar, con un close-up la cámara simula una perspectiva participante, la cual tomamos estando insertos en nuestra vida cotidiana, inmersos en nuestras cosas. Al terminar, con una toma extremadamente larga, la cámara simula una perspectiva externa, facilitada por nuestra capacidad de autoconsciencia reflexiva. Interesantemente, el movimiento continuo de la cámara sugiere que esas dos perspectivas no son radicalmente independientes la una de la otra: somos los únicos que podemos adoptar ambas perspectivas. El movimiento revela aún más la tensión creada por la yuxtaposición de estos dos puntos de vista (183). 
Esta yuxtaposición entre lo interno y lo externo se condice con un análisis de dos movimientos simultáneos realizados en las escenas mediante el zoom: un movimiento referido a Redmond y un movimiento referido al espectador. De Redmond a Fakenham y de Redmond a Lyndon tenemos el devenir de Redmond en estos otros yoes, pero asimismo, el espectador cuando viaja junto con los zoom in realizados, va desde su posición que observa a Barry y a los demás, hacia la posición de Redmond mismo, colocándose en su lugar. Los planos finales de los zooms colocan de manifiesto el lugar al que Redmond llega luego del movimiento. Sin embargo, además la cámara, su lente, ha hecho un movimiento que coloca al espectador en el hombro de Barry, permitiéndole observar desde sus mismos ojos. El zoom no sólo implica la expresión de un movimiento del protagonista hacia quienes observa, sino que involucra el movimiento del espectador hacia Redmond con el fin de que el primero comprenda el movimiento del protagonista. El primer movimiento propuesto desde el espectador hacia Redmond es la posibilidad de que dicho espectador comprenda el segundo movimiento a efectuarse desde Redmond hacia Fakenham y Lyndon. Diremos entonces ahora que el zoom, en tanto movimiento visual, técnico, es entonces aquí expresión de un movimiento del yo, y este movimiento implica dos movimientos en su interior: movimiento del espectador hacia el protagonista y movimiento del protagonista hacia otros personajes de la historia.

Kubrick, al plantearnos transitar del qué al cómo, al proponernos esta óptica presente en Barry Lyndon nos hace un guiño epistemológico, nos invita a suspender por un momento el contenido manifiesto de la realidad y profundizar en el modo en que ésta se presenta, se articula, se mueve y expresa. Ello no significa abandonar alguna de sus dimensiones, sino más bien abrir otra diferente que articule íntegramente a las demás, que las decodifique; significa ingresar a una matriz de interpretaciones, relaciones o estructuras. Esto es suspender por un instante a Redmond, a Fakenham, a Lady Lyndon, al espectador. Implica profundizar en el carácter del "y" que existe entre estos personajes y que se expresa en el movimiento del zoom que los conecta y pone en movimiento. Redmond " $y$ " Fakenham, Redmond " $y$ " Lyndon, Redmond " $y$ " el espectador. No se trata tanto de quien ve o de lo que es visto, sino de cómo quien ve llega a observar lo que es visto y como lo visto llega a ser visto por quien ve. El zoom se presenta como el modo, como aquella clave que posibilita un saber, que nos muestra una certeza en Redmond sobre la búsqueda de la libertad. Hegel nos invita a ingresar a la sala de proyección:

Si reflexionamos sobre esta diferencia, resulta que ni el uno ni el otro están sólo de modo inmediato en la certeza sensorial, sino que ambos lo están, a la vez como mediados; yo tengo esta certeza por otro, a saber, la cosa; y ésta, igualmente, está en la certeza por otro, a saber, yo ( 163$)$.

Podríamos pensar que Fakenham y Lyndon se nos aparecen inmediatamente, la mirada de Redmond nos arroja subrepticiamente hacia una verdad nueva; la cámara nos sorprende sin previo aviso colocándonos en el propio lugar de Redmond. Sin 
embargo, examinando el asunto como ya lo hemos hecho, ninguno de estos elementos son certezas en sí mismas, ni aparecen no-mediadas aunque la velocidad del zoom nos sugiera lo contrario. Estos elementos se encuentran mediados. El zoom mismo aquí funciona como la expresión de una mediación entre los yoes, como un conector lógico que posibilita el movimiento entre aquellos. Este zoom nos permite "un pasar al otro desde sí y desde lo otro y, a la vez, donde lo otro pasa a lo otro de sí desde sí y desde lo otro de sí mismo, o sea, un mero dinamismo (Werden) 'correspectivo' que rompe la finitud de las determinaciones" (Espinoza, Arte y religión en la Phänomenologie des geistes de Hegel a la luz de la Wissenschaft der logik 164-165). El ser, la verdad o la esencia no está en Redmond, ni en Fakenham o Lyndon, tampoco en el espectador; están en la mediación de todos ellos a través del movimiento propuesto por el zoom. Este movimiento nos muestra en un inicio un plano general que se nos aparece como la superficie y que en la medida que avanza al siguiente plano va entrando, ingresando, profundizando en una verdad diferente a la anterior, a la vez que la articula y la transforma, en donde "la unidad y la distinción se unifican. En esta unificación lo profundo emerge a la superficie, es más: se hace superficie” (Vitiello 182). Se trata justamente de "un zoom [que] conecta los dos [ámbitos] y proporciona un continuo entre el mundo personal de la figura y el mundo real que lo comprende, y del cual [Redmond] es parte" (Ebiri 5). El zoom, así como se dirige hacia un lugar en el movimiento unificador que propone, también plantea a la vez el lugar desde el cual proviene, es decir, se expresa desde un inicio en este zoom no sólo otro por conocer, sino paralelamente un yo que conoce, que acompaña el movimiento propuesto. Se invita a Redmond a viajar, se invita al espectador a viajar. En clave hegeliana, este zoom es un para nosotros:

Hegel nos muestra en la Fenomenología del espíritu su modo mismo de hacer filosofía, ese modo es su "y" (und) que técnicamente Hegel llamará für es ("para la”). [...]. Für es es lo lógico mismo (das Logische) que articula los otros referentes (an sich y für sich), es la articulación misma [...] que hace posible cualquier tipo de binomio dialéctico [...]. La "y" (und) del an und für sich, es decir, el "para la” (für es) es en el fondo siempre un "para nosotros" (für uns), un nosotros histórico que agencia la mediación (Espinoza, Repensando a Deleuze y el 'acontecimiento' desde lo 'lógico' de la Wissenschaft der logik de Hegel... En torno al devenir cosa 164-165).

Se anuda entonces el sentido de los dos movimientos ya planteados, el de Redmond y el del espectador. Así como Fakenham y Lyndon se expresan para un nosotros, que en un primer momento es ocupado por Redmond como observador, así también las escenas, subsumiendo a Redmond observando, se nos muestran para nosotros, como espectadores, y desde allí cobran sentido y realidad en tanto escenas. Pero el zoom también rompe la finitud de las determinaciones, es decir, las palabras de Kubrick referidas a la búsqueda de la libertad mediante un cómo se condicen con la idea de la mediación como movimiento tendiente más que a un escape, a una liberación, a 
una ab-solución. Aparece la cuestión del método en Hegel, que apunta no solamente a la aprehensión de lo real desde la mediación y el devenir, sino también como una posibilidad de liberar al hombre de lo que lo determina:

Método absolutamente ab-suelto, método en libertad o, mejor dicho, método que libera, expresión misma de la libertad ya de lo racional como de los pliegues de lo real. Método y libertad son sinónimos. Método es la expresión misma de la operatividad de la libertad (Espinoza, Hegel y el problema del método 69).

Nuevamente, más allá de la astucia, la ambición y el arribismo, el film nos propone una búsqueda de la libertad en Redmond, dejando atrás las determinaciones de cada contexto en el que se va insertando. No hay libertad en sí, sino más bien el zoom siempre expresa al final de su recorrido un modo de liberarse de las determinaciones actuales que, al ser habitada, plantea nuevas determinaciones. El ejército proveyó de sustento, pero le maltrató duramente, la vida de espía-mayordomo le permitió escapar de la miseria militar a una vida más cómoda, con un doble protectorado (ofrecidos por el Capitán Potzdorf y el Chevalier), pero le mantenía dependiente de sus mecenas; el juego le independizó y acercó a un nivel de vida lujoso y cercano a la nobleza, pero a condición de ser un errante y un tramposo; el matrimonio le llevó a la cúspide de lo social, mas, dado su origen, fracasó constantemente en la búsqueda de un título nobiliario. Kubrick nos habla del camino hacia la libertad, sin embargo en Barry Lyndon el inicio o el final de dicho camino no nos lleva a un lugar de plenitud para Redmond. Ello es porque la libertad es el camino mismo, que no posee ni inicio ni fin, camino que nuevamente en Hegel nos remite a lo lógico, al método:

El método nos lleva al problema del camino, un camino que a veces supone relatos, esto
es, los puntos tanto de inicio como de fin del camino propiamente tal. Pero otras veces
el método indica un camino en el caminar mismo donde los relatos son momentos
dinámicos de ese caminar; de esta manera es desde dónde Hegel estará pensando el
camino por excelencia de la libertad (Espinoza, Hegel y el problema del método 70).

En Barry Lyndon no hay algo así como un inicio o un final nítidos, se trata más bien de la selección de un momento y de su expresión fílmica. La película comienza con el duelo que da muerte al padre de Redmond y termina con el duelo al que lo reta Lord Bullingdon, comienzo y término anudados por un duelo que implican siempre el fin de un proceso y el inicio de otro, el punto de partida de un yo implica el tránsito de otro yo a algo diferente: la muerte. Los zooms constantemente nos hablan de este circuito también en el caso de la vida misma, esto lo hallamos expresado en los zooms outs utilizados en las escenas del juego de naipes entre Nora y Redmond y, posteriormente, en la del nacimiento de Bryan. En la primera escena aparece una escultura de un niño en el centro (figura 5), que al desplegarse el zoom muestra jugando a Redmond y a Nora, su primer amor (figura 6). En la segunda escena, el centro es ocupado por Bryan recién nacido (figura 7), y al alejarse el lente, aparece Redmond y Lady Lyn- 


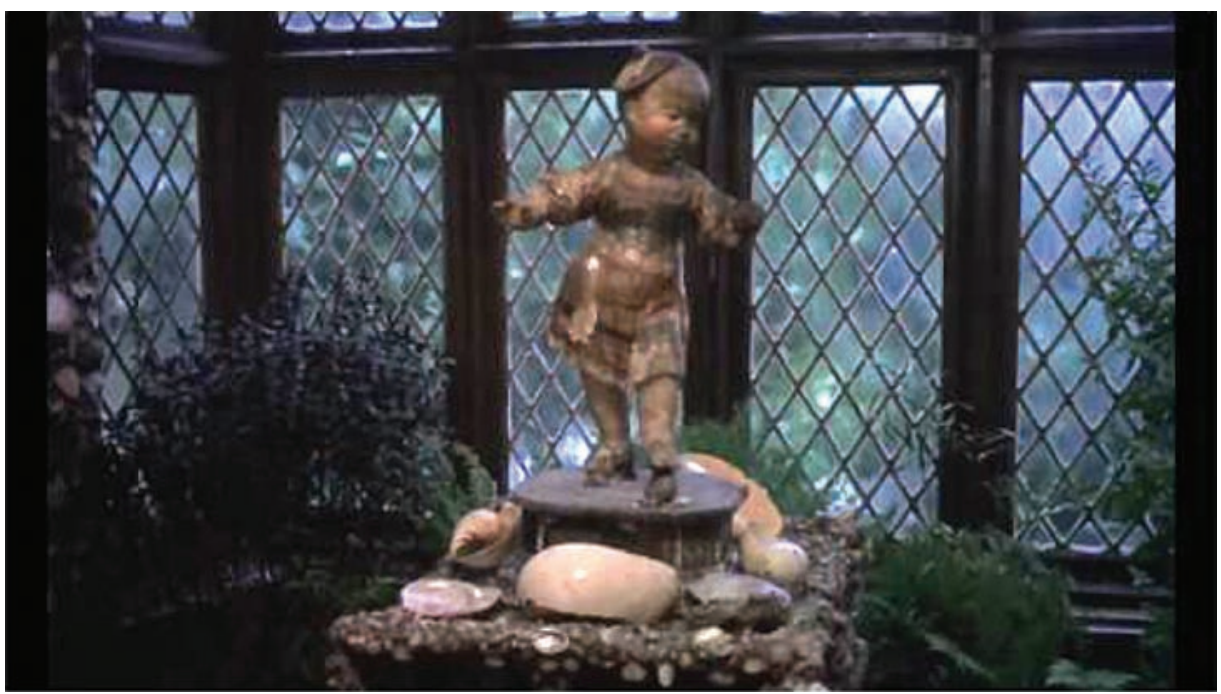

Figura 5. Una escultura de un niño, que representa la infancia de Redmond.

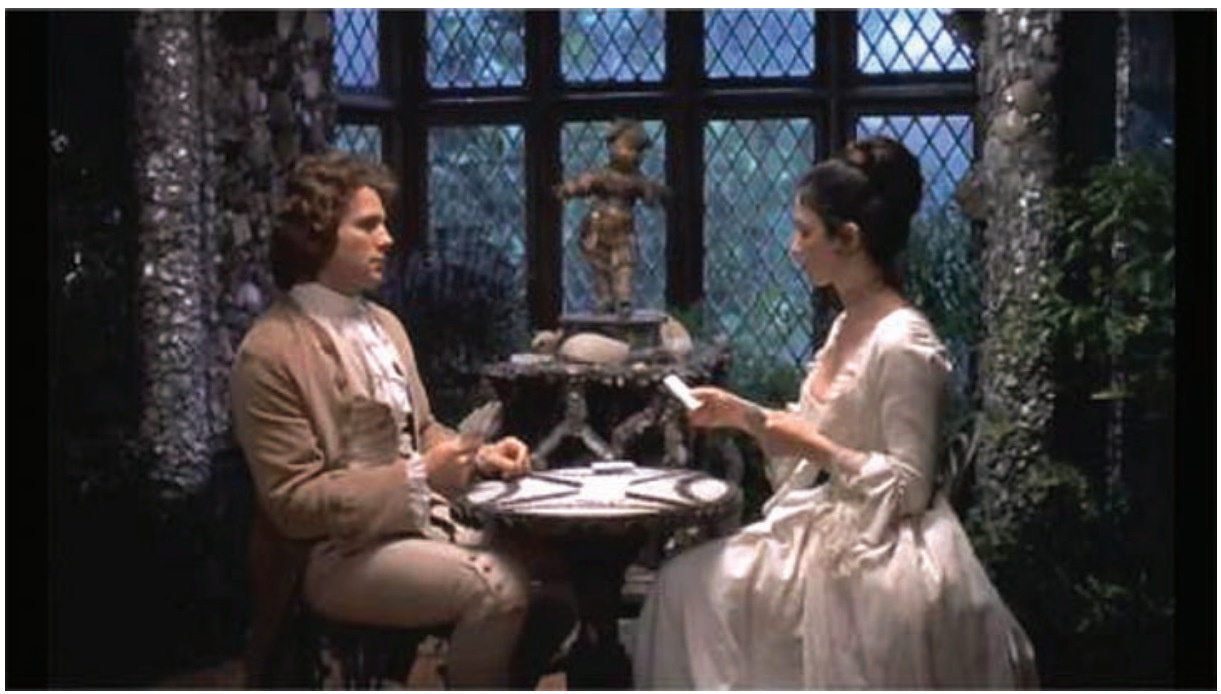

Figura 6. La niñez de Redmond es dejada atrás y el zoom retrocede para dar lugar al primer romance con Nora.

don acurrucándolo (figura 8). El niño ha devenido otro niño, Redmond ya no es el niño que juega al amor, el niño de la escultura al centro; Redmond deviene en padre, ahora a un costado de la escena, padre de un nuevo niño, su hijo; pero Redmond sigue siendo el niño luego del duelo con Lord Bullingdon, su madre le acompaña hasta la muerte; herido en la pierna no puede caminar, ha vuelto a la infancia, a ser un hijo. Son variados los ejemplos mediante los cuales Kubrick nos presenta la vida y la muerte, en tanto nacimiento y duelo, como momentos que el zoom también va 


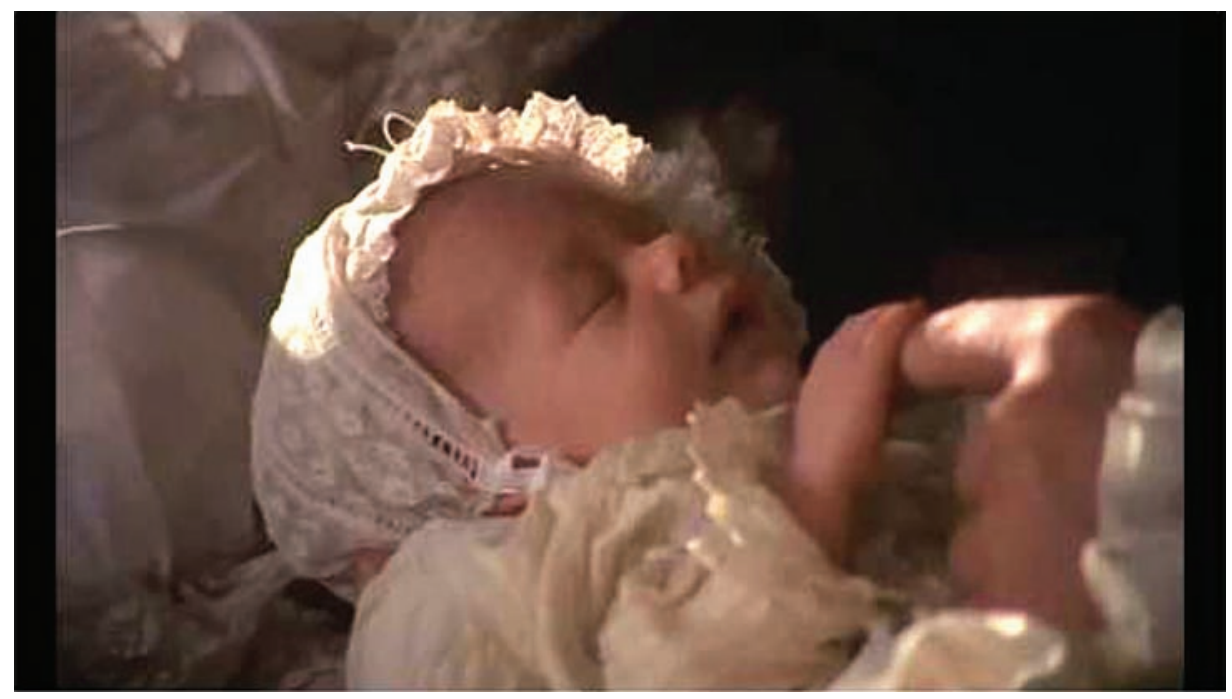

Figura 7. La escultura del niño es reemplazada aquí por el hijo de Redmond.

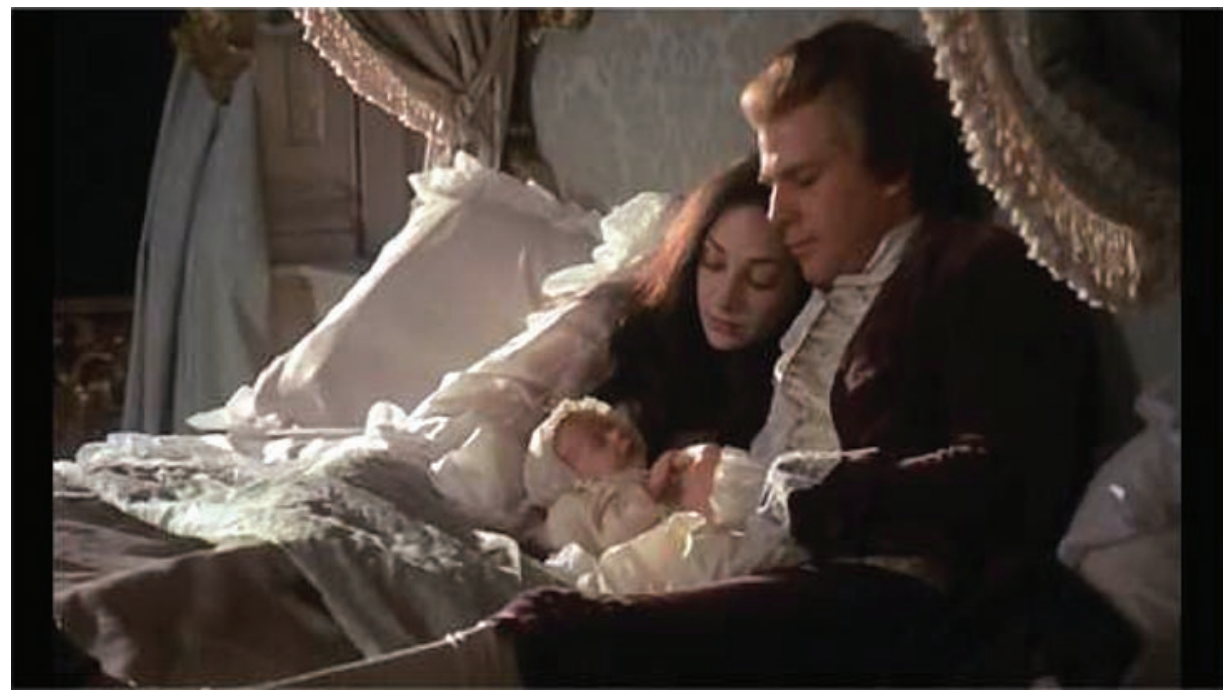

Figura 8. Redmond deja de ser hijo para devenir padre, el zoom ya no muestra un amor pasional de juventud, sino a un matrimonio bastante conveniente; Lady Lyndon reemplaza a Nora.

expresando y movilizando de fractales maneras. Más que un inicio y un final nos hallamos con una historia y un sistema de pasajes, tránsitos, quiebres, nudos; más que un camino son múltiples senderos interconectados que nos hablan de la libertad como posibilidad de recorridos a escoger, sin la primacía a priori de uno sobre otro. Como menciona el epílogo: "Fue durante el reinado de Jorge III que los personajes mencionados vivieron y altercaron. Buenos o malos, hermosos o feos, ricos o pobres, todos son ahora iguales"; a pesar de toda determinación, todos son ahora absueltos. 
Por otro lado, estos caminos nos hablan de la libertad en tanto implican una superación de y en los momentos en los que Redmond se encuentra, ambos zooms se mueven hacia un destino social diferente, en donde incluso algunos elementos de las escenas sugieren metafóricamente aquel pasaje: el lago y la pileta. Fakenham en el lago y Lady Lyndon tras la pileta mientan el agua, lo líquido como dimensión que confronta el terreno sólido desde el cual Redmond observa y se afirma, la seguridad de un tronco, la comodidad de una terraza. Lo líquido apela a que Redmond fluya y devenga en ello, el agua coloca la diferencia como destino del movimiento de un yo absuelto. La negatividad se muestra de aquella manera: se trata, según aquella metáfora, de una otredad líquida que, mediada por el zoom, dota a Redmond de un territorio. Más hegelianamente, de la mediación entre un Redmond y un no-Redmond:

\footnotetext{
Yo, éste yo, veo el árbol y afirmo al árbol como estando aquí; otro yo, empero, ve la casa y afirma que el aquí no es un árbol, sino, más bien, una casa. Ambas verdades tienen la misma acreditación, a saber, la inmediatez del ver, y la seguridad y la aseveración de ambas acerca de su saber; pero una desaparece en la otra.

Lo que no desaparece es el yo en cuanto universal, cuyo ver no es un ver el árbol ni un ver la casa, sino un simple ver que media a través de la negación de esta casa y demás, y al hacerlo es igualmente simple e indiferente frente a cualquier otro ejemplo, frente a la casa o el árbol. El yo es sólo algo universal, como el ahora, aquí o esto en general; sin duda, yo quiero decir íntimamente un yo singular, pero igual que no puedo decir lo que quiero íntimamente decir con ahora, o aquí, lo mismo pasa con $<<$ yo $>>$. [...], al decir yo, este yo singular, estoy diciendo, en general, TODOS los yos; cada uno de ellos es lo que yo digo; yo, éste, singular, yo (Hegel, 169).
}

Hay un estando aquí de Redmond en cada plano inicial, Redmond mirando desde la orilla, Redmond mirando desde la terraza. Su certeza es sobre el lugar social al que ha llegado, su saber es respecto a su afirmación en una nueva dimensión a la que ha transitado ya. Cuando aparece Fakenham, cuando aparece Lady Lyndon, esta afirmación es cuestionada: es posible un nuevo movimiento, es posible escapar, es posible ser otro yo. Redmond no desaparece, ya que no se trata de una negación contradictoria, sino una negación que constituye al mismo Redmond desde lo diferente, desde lo otro: Redmond es Redmond sólo en mediación con lo no-Redmond, es decir, con lo Fakenham y lo Lady Lyndon, los que, en sus momentos respectivos, se articulan como la negatividad de Redmond. No hay Redmond en-sí, sino sólo desde la negatividad ofrecida por estos otros yoes singulares. El zoom entonces es un simple ver de Redmond que media a través de la negación de su identidad, simple ver que media con Fakenham, que media con Lady Lyndon; zoom de mediación con su no-Redmond. Y más aún, el zoom es mediación de la negación de Fakenham, de Lady Lyndon y del espectador. Estos yoes son negados y devienen otros yoes a través del movimiento del lente. En Barry Lyndon, Kubrick nos propone un zoom que nos habla de la negatividad como motor del devenir, de la libertad, zoom lógico, zoom 
metódico. Al decir Redmond, este Redmond, estoy diciendo en general, TODOS los Redmond; Jonathan Fakenham y Lady Lyndon es lo que Redmond dice, y lo que yo, éste espectador singular, digo.

\section{Un epílogo kantiano: un Lord Lyndon en-sí.}

Sin embargo, este devenir inevitablemente encuentra un afán que pretende reinstalar la escisión, colocando en la mediación una imposibilidad, algo así como una determinación inamovible. Las filosofías de la escisión permanecen paralelamente a esta lógica hegeliana como momentos no superados de un devenir que las subsume. La separación kantiana entre fenómeno y noúmeno seguirá permaneciendo entre aquellos que ven en Hegel una ambición totalitaria de comprender el holos. Estará presente hasta nuestros días tanto en la filosofía como en diferentes disciplinas académicas, presente en nuestras instituciones sociales y en el quehacer tecnocientífico, en nuestra sexualidad, afectividad y vida cotidiana. Hegel abre dicha escisión hacia una posibilidad de absolución, sin embargo esta posibilidad nos habla también de la permanencia en nuestra sociedad de una escisión que se resiste por parte de nosotros a ser abandonada . Quizá sea Immanuel Kant quien, con anterioridad a Hegel, tematizó la escisión de manera consciente, crítica, pero finalmente reconociendo su dominio por sobre la razón. Kant reconoce los límites de aquella ante los conceptos de Dios, la Naturaleza y el Hombre, en tanto incognoscibles. La razón es insuficiente y lo que de estas nociones se desprenda estaría bajo el peligro constante de ser superchería. Kant comenta al respecto de la idea de Dios:

En efecto, todos los principios sintéticos del entendimiento son de uso inmanente;
pero para el conocimiento de un ente supremo se necesita un uso trascendente de
ellos, para el cual no está preparado nuestro entendimiento [...]. Sea cual fuere
el modo como el entendimiento haya llegado a este concepto, no puede hallarse
analíticamente en él la existencia del objeto mismo porque el conocimiento de la
existencia del objeto consiste precisamente en que éste está puesto en sí mismo fuera
del pensamiento (278).

De esta manera, si bien Kant pretende inicialmente superar la escisión, termina reconociendo el ámbito del nóumeno por fuera de lo cognoscible, y por tanto como lugar de lo en-sí, que provee de excusa a cualquier totalitarismo como intérprete privilegiado del fundamento social. Dejar abierta la puerta para lo en-sí es hacer posible la mediación mística, divina, poética o trascendente de un humano más allá de lo humano que puede conocer aquello que lo determina. Y en Barry Lyndon encontramos un claro ejemplo hacia el final del film referido a la pretensión de insertar en el devenir un fundamento último de la realidad, un pivote de gravitación necesario que daría sentido a la vida pasada y futura, propuesto como un deber ser. 
Es la madre de Redmond, que reaparece al final de la vida noble de su hijo, quien operará kantianamente instalando una necesidad, un imperativo, el fundamento que permitirá a Barry escapar ,finalmente, al flujo constante de la realidad y determinar su destino haciendo innecesarias las relaciones que posee con los demás. Su madre dirá, respecto a Lady Lyndon:

¿Pero y si se cansara de mi indomable Redmond y de sus modales irlandeses? ¿O si muriera? ¿Qué futuro tendrían entonces mi hijo y mi nieto? Tú no tienes ni un solo penique y sin su firma no puedes hacer nada. A su muerte la fortuna pasaría a Lord Bullingdon que te profesa muy poco afecto. Podrías verte pobre mañana y mi querido Bryan quedaría a merced de su hermanastro. ¿Quieres oír mi opinión? Sólo hay un modo de que tú y tu hijo estén a cubierto. Tienes que obtener un título. No descansaré hasta que te vea llamarte Lord Lyndon (Barry Lyndon).

El título nobiliario, devenir un Lord aparece como el único modo. El devenir constante de Redmond a través de sus mediaciones intenta ser fijado por su madre, de una vez y para siempre por un en-sí que le permitirá determinar totalmente su ser y restar importancia al acontecimiento. El narrador, consciente de las implicancias, afirma: "La lucha por este título fue la empresa más desdichada de Barry". En efecto, nunca antes Redmond se afanó tanto por la transformación completa de su ser como cuando pretendió adquirir el título de Lord Lyndon, y es que, a diferencia de las mediaciones anteriores que emergen contingentemente, en un camino de posibilidades abiertas, no contradictorias, yuxtapuestas, este ser un Lord es enunciado como el "único modo", como "un tener que", como el freno del devenir de su historia. Y vemos que la historia como devenir se rebela ante la obtusa intención de fijarla desde la necesidad. Redmond fracasa completamente en su intención, provocando la ruina económica, que se concatena a una red de sucesos: la ira de su hijastro, el desprecio social, la melancolía de Lady Lyndon, la pérdida de su pierna, hechos que dibujan un final trágico. Redmond, confiando en su madre, recoge sus consejos luego de una vida construida de manera solitaria. La inserción del en-sí materno como ley, en tanto exterioridad, no encuentra más que un fracaso en este film, que consigo arrastra la clausura y el derrumbe de aquella libertad en devenir.

\section{Referencias}

Aula de cine. Hoja informativa $N^{\circ}$ 17, 2004. <http://www.aulas.ulpgc.es/cine/ historial/017-Barry\%20Lyndon.pdf $>$. Sitio web.

Barry Lyndon. Kubrick, Stanley, dir. Act. Ryan O'Neal, Marisa Berenson. 1975. Medio fílmico.

Ciment, Michel. Kubrick: the definitive edition. USA : Faber and Faber, 2003. Medio impreso. 
---. Kubrick: edición definitiva. Trad. Esperanza Martínez y Leonor Celorio MéndezTrelles. Madrid: Ediciones Akal, 2000. Medio impreso.

--. Kubrick on Barry Lyndon: an interview with Michel Ciment. <http://www.visualmemory.co.uk/amk/doc/interview.bl.html>. Sitio web.

Ebiri, Bilge. Barry Lyndon: The shape of things to come. <http://www.visual-memory. co.uk/amk/doc/0026.html>. Sitio web.

Espinoza, Ricardo. "Arte y religión en la Phänomenologie des geistes de Hegel a la luz de la Wissenschaft der logik...”. Daimón, Revista de Filosofía 43 (2003). 71-91. Medio impreso.

---. "Hegel y el problema del método". Revista Estudios Hegelianos 1 (2012). 67-77. Medio impreso.

---. 'Repensando a Deleuze y el 'acontecimiento' desde lo 'lógico' de la Wissenschaft der logik de Hegel... En torno al devenir cosa”. Hegel. La transformación de los espacios sociales. Ed. Ricardo Espinoza. Concón: Midas, 2012. 155-175.

Falsetto, Mario. Stanley Kubrick: a narrative and stylistic analysis. USA : Praeger Publishers, 2001. Medio impreso.

Hegel, Georg Wilhelm Friedrich. Fenomenología del espíritu. Trad. Manuel Jiménez Redondo. Valencia: Pre-Textos, 2006. Medio impreso.

---. Fenomenología del espíritu. Trad. Antonio Gómez Ramos. Madrid: Abada, 2010. Medio impreso.

Kant, Immanuel. Crítica de la razón pura. Buenos Aires: Losada, 1938. Medio impreso. Photographing Stanley Kubrick's Barry Lyndon. American Cinematographer 57, ㅇ3 (1976). <http://www.visual-memory.co.uk/sk/2001a/bl/page1.htm>. Sitio web.

Pliatska, Chris. "The absurd and Barry Lyndon". The philosophy of Stanley Kubrick (2007). 183-200. Medio impreso.

Popper, Karl. La sociedad abierta y sus enemigos. Barcelona: Paidos, 2010. Medio impreso.

Stanley Kubrick: a life in pictures. Harlan, Jan, dir. Act. Tom Cruise, Nicole Kidman, Jack Nicholson, <http://www.youtube.com/watch?v=FR-loS9MHww $>$. Sitio web.

Vitiello, Vincenzo. "Hegel. Lógica/realidad: la superficie y el fondo". Hegel. La transformación de los espacios sociales. Ed. Ricardo Espinoza. Concón: Midas. 177202. Medio impreso.

Voegelin, Eric. “Sobre Hegel: un estudio de brujería”. Foro interno 10 (2010). 155-197. Medio impreso. 